\title{
A Two-Phase Model Based on SVM and Conjoint Analysis for Credit Scoring
}

\author{
Kin Keung Lai, Ligang Zhou, and Lean Yu \\ Department of Management Sciences, City University of Hong Kong, Hong Kong \\ \{mskklai,mszhoulg, msyulean\}@cityu.edu.hk
}

\begin{abstract}
In this study, we use least square support vector machines (LSSVM) to construct a credit scoring model and introduce conjoint analysis technique to analyze the relative importance of each input feature for making the decision in the model. A test based on a real-world credit dataset shows that the proposed model has good classification accuracy and can help explain the decision. Hence, it is an alternative model for credit scoring tasks.
\end{abstract}

Keywords: support vector machines, conjoint analysis, credit scoring.

\section{Introduction}

For financial institutions, the ability to predict if a loan applicant or existing customer will default or not is crucial, and an improvement in prediction accuracy can help reduce losses significantly. Most statistic methods and optimization techniques and some new approaches in artificial intelligence have also been used for developing credit scoring models. A comprehensive descriptions of methods being used in credit scoring can be found in a recent survey [1].

Each method has its advantage and disadvantage, so it is difficult to find one model that can perform consistently better than other models in all circumstances. For the measure of classification accuracy, AI technologies can perform better than traditional methods; however, their black-box property make it difficult for the decision makers to use them with adequate confidence. In this study, we introduce a LSSVM [2] approach with the radial basis function (RBF) kernel and adopt an approach based on the principle of design of experiment (DOE) to optimize the parameters [3. In addition, the conjoint analysis method is used to calculate the relative importance of each input feature for credit risk evaluation.

The rest of this paper is organized as follows. Section 2 illustrates the basic concepts in LSSVM and the main process in conjoint analysis and describes our method. In Section 3, we use a real-world dataset to test the proposed method and analyze the results with conjoint analysis. Section 4 provides a conclusion about this study. 


\section{Two-Stage Model Based on SVM and Conjoint Analysis}

Given a training dataset $\left\{x_{k}, y_{k}\right\}_{k=1}^{N}$, we can formulate the LSSVM model in feature space as following [2]:

$$
\min _{w, b, \xi} \zeta(w, \xi)=\frac{1}{2} w^{T} w+\frac{C}{2} \sum_{k=1}^{N} \xi_{k}^{2}
$$

Subject to: $y_{k}\left[w^{T} \varphi\left(x_{k}\right)+b\right]=1-\xi_{k}, k=1, \ldots, N$

The classifier in the dural space takes the form:

$$
y(x)=\operatorname{sign}\left[\sum_{k=1}^{N} \alpha_{k} y_{k} K\left(x, x_{k}\right)+b\right]
$$

Where the $\alpha_{k}$ is the Lagrange multiplier, in this study, we chose kernel function to be Radial-basis function (RBF): $K\left(x, x_{k}\right)=\exp \left(-\left\|x-x_{k}\right\|^{2} / \sigma^{2}\right)$.

In the above LSSVM model, there are two parameters to be determined, $C$ and $\sigma$. A method inspired by DOE proposed by Staelin [3] can reduce the complexity sharply, relative to grid search method. The main steps of this approach are as follows:

1. Set initial range for $C$ and $\sigma$ as [C_min, C_max], [D_min, D_max],iter $=1$;

2. While iter $\leq$ MAXITER do

2.1. According to the pattern as shown in Figure 1, find the 13 points in the space [(C_min, D_min), (C_max, D_max)], set C_Space=C_max-C_min, D_Space= D_max-D_min;

2.2. For each of the 13 points which have never been evaluated, carry out the evaluation: set the parameters according to coordinate of this point, run LSSVM via $k$-fold cross validation, the average classification accuracy is the performance index of this point;

2.3. Choose the point $\mathrm{p} 0(\mathrm{C} 0, \mathrm{D} 0)$ with best performance to be the center, set new range of the searching space. C_Space= C_Space/2, D_Space=D_Space/2. If the rectangle area with $(\mathrm{C} 0, \mathrm{D} 0)$ as the center and C_Space, D_Space as width and height exceeds the initial range, adjust the center point until the new search space is contained within the [(C_min, D_min),(C_max, D_max)].

2.4. Set new searching space, C_min $=$ C0-C_Space $/ 2$, C_max $=$ C0 + C_Space $/ 2$, D_min=D0-D_Space/2, D_max=D0+D_Space $/ 2$, iter $=$ iter +1 ;

3. Return the point with best performance, use its best parameters to create a model for classification.

Conjoint analysis is a technique with wide commercial uses, such as prediction of profitability or market share for proposed new product, providing insights about how customers make trade-offs between various service features, etc. For an evaluated applicant with feature $x$, its utility can be defined by the value $\sum_{k=1}^{N} \alpha_{k} y_{k} K\left(x, x_{k}\right)+b$ from the LSSVM model, the larger is this value, the less is the possibility of its default. All the tested applicants can be ranked by their utility. Then the part-worth model is selected to be the utility estimation model 


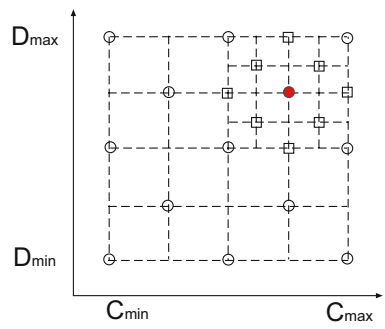

Fig. 1. Sketch of two iterations search with method based on DOE

because of its simplicity and popularity. We choose multiple regression method to estimate the part-worth, and ranking order of the applicants as the measurement scales for dependent variables. The decision manager, are concerned about not only the classification accuracy of the model but also the average importance of each attribute which can be measured by the relative importance of attribute in conjoint analysis. For each model, we can calculate the relative importance of attribute $i$ as the following formula:

$$
I_{i}=\frac{\max P_{i j}-\min P_{i j}}{\sum_{i=1}^{n}\left(\max P_{i j}-\min P_{i j}\right)} \times 100 \% \quad j=1, \ldots L_{i}
$$

Where $P_{i j}$ is the part-worth of level $j$ for attribute $i, L_{i}$ is the number of levels for attribute $i, n$ is the number of attributes.

\section{Empirical Study}

A real world data set German dataset from UCI is used. it consists of 700 good instances and 300 bad ones. For each instance, 24 input variables described 19 attributes. 5-fold cross validation is used to evaluate the performance of each group of parameters setting for the LSSVM model. We set MAXITER $=5$, searching space of $\log _{2} C$ is $[-5,15], \log _{2} \sigma$ is $[-15,5]$ and finally get the optimal parameters $C=2^{7.5}, \sigma=2^{5.0}$. Then we use 10 -fold cross validation to test the efficiency of this model and five repetitions are conducted to reduce the stochastic variability of model training and the testing process. The results were also compared with other methods on the same dataset shown in Table 1.

The proposed method can provide high classification accuracy, but a major deficiency of the model is the difficulty in explaining the role of the input features for making the decision. We conjoint analysis to calculate the relative importance of each feature after ranking the utility of the testing sample. For the 10-fold cross validation running, there are 10 group testing samples; Figure 2 illustrate the average relative importance of each feature of the 10 group testing samples for the LSSVM+DOE model. From this figure, we can see that only three features of the applicants' exceed $8 \%$. Although some of the features have less importance, they all contribute to the decision in the LSSVM+DOE model. 
Table 1. Results from different methods on the German credit data set

\begin{tabular}{lcc}
\hline Methods & Classification accuracy (\%) & Std. (\%) \\
\hline SVM+Grid Search & \\
SVM+Grid Search + F-score & & 3.86 \\
SVM+GA $^{a}$ & 76.00 & 4.03 \\
MOE $^{b}$ & 77.50 & 3.97 \\
$\mathrm{RBF}^{b}$ & 77.92 & - \\
$\mathrm{MLP}^{b}$ & 75.64 & - \\
$\mathrm{LVQ}^{b}$ & 74.60 & - \\
$\mathrm{FAR}^{b}$ & 73.28 & - \\
LS-SVM+DOE $^{b}$ & 68.37 & - \\
\hline
\end{tabular}

${ }^{a}$ Results from [4], ${ }^{b}$ results from [5].

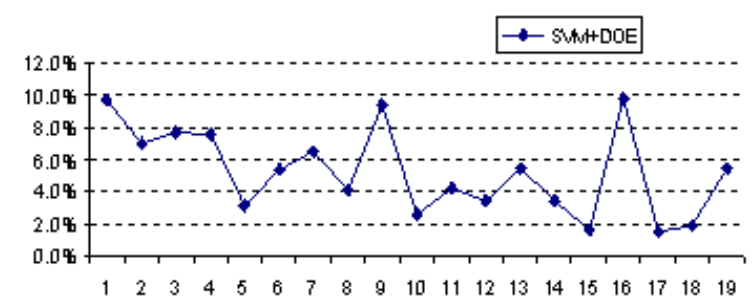

Fig. 2. Relative importance of features for German dataset

\section{Conclusion}

This study proposes a two-phrase model for credit scoring. The parameters of the LSSVM model are optimized by a searching procedure inspired by Design of Experiment. Then the decision from the LSSVM model is estimated by the conjoint analysis method. The relative importance of attributes derived from conjoint analysis provide the decision makers with some idea about what features of the applicant are of importance for the model and whether the decision is consistent with their past experience. The results show that the proposed model has good classification accuracy and, in some degree, can help the decision makers to explain their decision.

\section{References}

1. Thomas, L.C.: A Survey of Credit and Behavioural Scoring: Forecasting Financial Risk of Lending to Consumers. International Journal of Forecasting 16 (2000) 149-172

2. Suykens, J.A.K., Gestel, T.V., Brabanter, J.D., Moor, B.D., Vandewalle, J.: Least Squares Support Vector Machines. World Scientific, Siningapore (2002)

3. STAELIN, C.: Parameter Selection for Support Vector Machines. Tech. Rep., HPL-2002-354 (R. 1), HP Laboratories Israel, (2003) 
4. Huang, C.L., Chen, M.C., Wang, C.J.: Credit Scoring with a Data Mining Approach Based on Support Vector Machines. Expert Systems with Applications (2006) doi:10.1016/j.eswa.2006.1007.1007

5. West, D.: Neural Network Credit Scoring Models. Computers \& Operations Research 27 (2000) 1131-1152 